\title{
La Enseñanza del Inglés como Segunda Lengua*
}

\author{
Manuel J. Mederos*
}

\begin{abstract}
Resumen
Este artículo presenta marcos teóricos de origen socio-cultural y lingüístico-cognoscitivo que han influido en la enseñanza de inglés como segundo idioma. Los paradigmas conceptuales que se presentan establecen que el idioma es el instrumento principal de la enseñanza y el medio primordial para crear relaciones interpersonales y establecer comunicación. Debido a que, en la mayoría de los casos, la enseñanza del idioma se lleva a cabo en el aula escolar, los propósitos del contexto comunicativo se relacionan con el aprendizaje de los conceptos académicos. Este artículo propone una guía para el desarrollo de unidades curriculares para la enseñanza del idioma a través del contenido de la ciencia. Los elementos de esta guía se fundamentan en algunos de los factores socio-culturales y académico-cognoscitivos que se discuten dentro de los marcos teoréticos que se presentan en este artículo.
\end{abstract}

Palabras clave: enseñanza del inglés; segunda lengua; unidades curriculares; ciencia

\section{The teaching of English as a second language}

\begin{abstract}
This article presents socio-cultural and linguistic-cognoscitive theoretical frameworks which have influenced the teaching of English as a second language. The conceptual paradigms proposed in this work point out language as the teaching main instrument and as the fundamental mean to build interpersonal relationships and communication. As language teaching is usually done within the classroom the purposes of the communicative context are linked to the learning of academic concepts. This article suggests a guideline for the development of language teaching curricular units though contents related to science. The elements of this guideline are based on some of the socio-cultural and academic-cognoscitive factors discussed in the theoretical frameworks this article presents.
\end{abstract}

Key words: Teaching of English; Second Language; Curricular Units; Science

* Este trabajo forma parte de la investigación "Propuesta de programa para el desarrollo de la comunicación oral en inglés específico, a partir del uso de la inteligencia múltiple”.

Licenciado en Educación. Msc. en Educación Universitaria. Sede universitaria municipal de Ciencias Médicas San Antonio de los Baños. valiente@uci.cu 


\section{Introducción}

Por tradición, la enseñanza de idiomas en Estados Unidos y en otros países de habla inglesa, se basa mayormente en nociones de pedagogía y lingüística. Podemos trazar el origen de la metodología de la enseñanza de segundos idiomas a una combinación de marcos teóricos de lenguaje y enseñanza.

El estructuralismo de Saussure, el descriptivismo norteamericano, la gramática generativa (Chomsky, 1965) y el método de codificación cognoscitiva (Carroll, 1965) son algunas de las nociones teoréticas que orientan tanto la metodología de la enseñanza, como el desarrollo del currículo y materiales, la evaluación y la medición.

La metodología del siglo veinte se origina en torno a nociones teoréticas propuestas por Saussure que establecen que el idioma representa un grupo limitado de combinaciones de fonemas. Esta teoría fue expandida por la psicología del comportamiento, la cual establece que el idioma se adquiere formando hábitos, incorporando una sintaxis a los fonemas por medio del uso de unas reglas predeterminadas, y combinando las categorías gramaticales. El modelo de proficiencia de idioma de Harris (1969) y exámenes tales como el "Comprehensive English Language Test (CELT)" y el "Test of English as a Foreign Language (TOEFL)" asumen que la proficiencia de un idioma consiste de estructuras individuales que comprenden dos dimensiones: I) destrezas del idioma (escuchar, hablar, leer y escribir) y 2) fonología/morfología, gramática, vocabulario y fluidez en general. Estos fundamentos se utilizaron para desarrollar la metodología audiolingüe que aísla los patrones lingüísticos de la conversación natural, enfoca la estructura y la repetición, y donde el contexto y el significado no tienen gran importancia. Al tomar en cuenta problemas de naturaleza conceptual surge la necesidad de modificar los marcos teóricos mencionados anteriormente. Los siguientes dilemas son algunos ejemplos que motivaron el cambio: I) las destrezas no están aisladas por estructuras lingüísticas (por ejemplo, la secuencia del tiempo puede ser señalada por verbos, adverbios, eventos y expresiones); 2) los exámenes miden diferentes clases de conocimiento (son de naturaleza integrativa); 3 ) 
los exámenes miden un trasfondo académico general; y 4) las partes de un examen tienen una correlación alta, es decir, las partes de un examen no miden habilidades aisladas (Oller, 1979; Spolsky, 1973).

La gramática generativa de Chomsky (1965) incorpora la creencia de que los patrones lingüísticos surgen del conocimiento adquirido y son almacenados en nuestras mentes. De acuerdo con Chomsky los individuos poseen un aparato de idiomas (LAD), que es de naturaleza innata y universal, el cual permite el aprendizaje de cualquier idioma. Como resultado de las ideas promulgadas por Chomsky la metodología de la enseñanza de idiomas se modifica otra vez para reflejar el concepto de ejecución.

El modelo de Canale y Swain (1980) incorpora el concepto de ejecución comunicativa de Chomsky pero añade otras tres competencias: sociolingüística, estratégica y la de conversación. Krashen (I98I) recoge en su modelo conceptual las transformaciones discutidas anteriormiente, pero desarrolla y añade el concepto de insumo de información comprensible (comprehensible input) y un componente cognoscitivo, el monitor, como elementos esenciales del aprendizaje. La metodología de la enseñanza del inglés como segundo idioma conocida como el Método Natural utiliza el modelo de Krashen (Natural Approach, Krashen and Terrel, 1983) como marco conceptual. Por otra parte, la teoría de Cummins (I979) recoge las ideas ya mencionadas y añade otros tres conceptos: la interdependencia entre el idioma nativo y el segundo idioma, las destrezas básicas para la comunicación interpersonal (BICS) y la proficiencia cognoscitiva y académica del idioma (CALP). La revisión del modelo de Cummins (Cummins y Swain, 1986) toma en consideración factores sociolingüísticos que enfatizan la importancia del contexto para facilitar la comunicación, el aprendizaje y el entendimiento. La evolución de los paradigmas descritos se vierte en un torrente de controversias que generan incógnitas, tales como: ¿Existe un periodo crítico para el aprendizaje de un segundo idioma? ¿Hay diferencias entre el aprendizaje de un primer y un segundo idioma?

Las ideas de Bley-Vroman (1988) y de White (1990) arrojan luz para la explicación de estos paradigmas. Bley-Vroman (I988) 
establece que los adultos poseen habilidades generales de la adquisición de idiomas siempre y cuando exista un ambiente apropiado. Por su parte, White (1990) indica que cuando se aprende un segundo idioma (L2) el estudiante tiene que ajustar los parámetros establecidos por su primer idioma (LI). Para ajustar los parámetros del primer idioma se requiere conocimiento de los parámetros del segundo idioma que se encuentran en el aparato de adquisición de idiomas (LAD).

\section{Desarrollo}

Las teorías que establecen la proficiencia cognoscitiva y académica del idioma y las que afirman que el aprender un segundo idioma requiere de la modificación de los parámetros del primer idioma, junto a las teorías que reconocen la influencia del ambiente y enfatizan la importancia del contexto para promulgar el aprendizaje y entendimiento del idioma, son las que han hecho posible la transformación de los marcos conceptuales para la enseñanza del inglés como segundo idioma.

Las teorías socio-pedagógicas reconocen que el aula escolar es el ambiente social más relevante para el estudiante. La interacción social que surge en el ambiente académico natural del aula es esencial para que los estudiantes intercambien conocimientos e interactúen en su idioma. Es en el aula escolar donde los estudiantes pasan la mayor parte de su tiempo haciendo lo que se espera de ellos: "aprender". El aprendizaje significativo de los estudiantes depende en gran parte del conocimiento previo. El esquema mental previo es la base para el procesamiento y el entendimiento (Rummelhart, 1980) del nuevo conocimiento. Durante el proceso de aprendizaje de conceptos de una materia dada el estudiante utiliza los esquemas desarrollados en aprendizajes anteriores para construir nuevos esquemas mentales o ampliar los ya existentes. Esto podría considerarse cierto tanto en el aprendizaje del contenido de las materias como en el aprendizaje del idioma. En el caso de un estudiante que ingresa en el nivel preescolar, el conocimiento previo depende de las bases sentadas en el hogar. Respecto a los estudiantes en otros niveles escolares el conocimiento previo representa sus experiencias adquiridas a través 
de las actividades educativas que incluyen contenido, destrezas de las materias, de pensamiento y del idioma.

El estudiante que aprende, tanto un idioma como una materia académica, necesita una representación mental de la relación entre su conocimiento previo y los atributos del conocimiento que va a adquirir. Durante el proceso de aprendizaje, los estudiantes necesitan tanto esquemas apropiados del idioma como de las materias académicas. Estos esquemas representan la base del aprendizaje. El aprendiz de un segundo idioma necesita activar el "esquema" apropiado para incorporar el nuevo conocimiento. Si asumimos que el estudiante trae consigo el esquema apropiado corremos el riesgo de encontrar que el esquema del idioma, del ambiente o de la cultura del estudiante o la falta de éstos interfiera con el entendimiento de la materia. Asumir que el estudiante tiene un conocimiento básico previo de la materia puede resultar en otro tipo de interferencia con el aprendizaje. El proceso de interpretación de la materia, del idioma o de ambos puede ser interrumpido por la falta de esquemas apropiados. Los procesos de recordar la información necesaria, de organizar las ideas, de interpretar, de hacer inferencias, de identificar y de aplicar conceptos pueden ser interrumpidos a causa de la falta de esquemas apropiados.

Estas teorías con bases cognoscitivas también asumen que el entendimiento de la materia requiere un dominio básico del idioma. Cuando el estudiante no posee las destrezas básicas del idioma, la instrucción debe proveer los estímulos requeridos para el entendimiento a través del contexto. El contexto de enseñanza debe incluir actividades que incorporen esquemas que provean estímulos académicos, lingüísticos, culturales y sociales apropiados y que sean adecuados para la edad y el desarrollo físico y mental del estudiante.

La enseñanza de un segundo idioma requiere el desarrollo de actividades basadas en experiencias concretas que activen los esquemas de conocimiento de las materias de estudio y del idioma para promulgar tanto la adquisición del segundo idioma como el aprendizaje de conceptos de ciencia. En otras palabras, este enfoque multidisciplinario requiere la integración sistemática de las actividades para la enseñanza del inglés como segundo idioma con 
actividades concretas para enseñar ciencia, o ambas, y en donde los estudiantes usen materiales manipulativos.

\section{Métodos Interactivos Para la Enzeñanza de un Segundo Idioma}

Los métodos interactivos para la enseñanza de un segundo idioma reconocen que la enseñanza de un segundo idioma es para el uso con y a través de una comunidad o grupo que habla el segundo idioma. En el contexto escolar la comunidad está en la escuela, en el salón de clases, en la vecindad o en la combinación de todas o alguna de las anteriores. Los estudiantes y el maestro, y en algunos lugares (como en ciertos estados de la Nación) los miembros del hogar y de la vecindad, forman parte de las personas que hablan el segundo idioma.

El uso del segundo idioma entre los estudiantes y los miembros de la comunidad (en la escuela o el vecindario) es mayormente para la comunicación entre sí y la interacción social. En el aula escolar es donde se enfatiza el uso del segundo idioma para el aprendizaje a través de las materias. Por esto, al diseñar actividades interactivas para la enseñanza del segundo idioma se debe tomar en cuenta que:

a. el idioma es el vehículo principal para la instrucción y el aprendizaje, esto es, el idioma relaciona lo que se dice (contenido lingüístico) con el mecanismo que se usa para decirlo (expresión lingüística);

b. el idioma juega un papel esencial en la interacción social; el idioma es un mecanismo para establecer relaciones sociales y aprender sobre el mundo en general y en el aula escolar;

c. el idioma esta intrínsicamente relacionado con el contenido utilizado en la instrucción; el segundo idioma es un medio para aprender el material académico;

d. el esquema-contexto, experiencias y conocimiento previo juegan un papel esencial en el aprendizaje del nuevo conocimiento; elementos de la cultura del hogar y del primer idioma forman parte del esquema previo del aprendiz; el idioma del hogar porta parte de la cultura del estudiante; el estudiante se puede beneficiar de la transferencia de destrezas: dentro del esquema 
que el estudiante posee al entrar al ambiente de aprendizaje se encuentran destrezas que se pueden transferir, como por ejemplo, destrezas de lectura del LI al L2 y de visualización espacial;

e. la comunicación, interacción y el desarrollo de conceptos en el salón de clases requiere mayormente de destrezas de proficiencia académico-cognoscitivas; por ejemplo, la ciencia y la matemática requieren conocimiento de vocabulario y de símbolos específicos de las ciencias y las matemáticas, tales como: volumen, por, tabla, exponente,,,$+- \mathrm{x}$;

f. La planificación de estas actividades requiere: que el profesor sepa identificar las necesidades de aprendizaje de los estudiantes, pues la asimilación de los contenidos de la enseñanza va a depender de la necesidad real que tenga el estudiante de aprender un contenido dado relacionado con el dominio del idioma o de la ciencia:

- la coordinación entre los maestros de segundo idioma con los maestros de la materia o contenido,

- el análisis de las necesidades del aprendiz: lingüísticas, interpersonales, socioculturales y académicas,

- la identificación y descripción del ambiente académico del aprendiz,

- la descripción del ambiente comunicativo en el hogar y la escuela,

- la selección de materias y del currículo apropiado,

- la identificación de temas y capítulos adecuados en el currículo "regular" para el desarrollo de las actividades,

- la adaptación del currículo a través del desarrollo de actividades interactivas que reflejen la integración del contenido con el segundo idioma,

- el desarrollo de actividades interactivas que reflejen el esquema de conocimiento lingǘstico, académico y social previo del estudiante,

- el desarrollo de actividades que reflejen el trasfondo cultural del estudiante; 


\section{Integración de la Enseñanza del Inglés como Segundo Idioma con Otras Disciplinas}

La comunicación en el salón de clases se lleva a cabo en gran parte a través de las materias académicas. Por lo tanto, el aprendizaje del idioma debe ser restructurado de acuerdo con el contexto académico. Esta forma de enseñanza del idioma requiere la selección de una materia que sea favorable para aprender el segundo idioma.

Hay materias que son tradicionalmente bien dependientes del idioma. Cuando la materia depende grandemente del idioma puede surgir una interferencia, causada por la falta del idioma, que afecte la calidad del esquema que el aprendiz tenga disponible. Una lección "tradicional" de estudios sociales sobre el tema de Gobierno en los EE.UU. (US) puede requerir el uso de un nivel de idioma muy avanzado para el estudiante. Además, es posible que el aprendiz no esté familiarizado con la forma en que se llevan a cabo las decisiones políticas en los EE.UU. Esto aumenta la interferencia causada por la falta de esquemas apropiados de conocimiento sobre la cultura y el ambiente. Aunque el estudiante tenga parte del conocimiento previo (esquema académico) requerido para entender el concepto "gobierno", la interferencia por la falta de esquemas socio-políticos apropiados es tal que limita la cantidad y calidad de la adquisición de insumos comprensibles tanto de la materia como del idioma. Por lo tanto, es esencial que se seleccione cuidadosamente el contenido de la materia que planificamos integrar con la enseñanza de inglés como segundo idioma.

\section{La Integración de Inglés como segundo idioma con la Ciencia}

La enseñanza de los principios científicos en forma concreta es un enfoque que permite tanto la adquisición de conceptos científicos como del segundo idioma. A través de actividades concretas e interactivas la ciencia provee una oportunidad para convertir las experiencias académicas a lenguaje. Las actividades interactivas proveen una oportunidad para la interacción entre los estudiantes dentro de un contexto comunicativo. El lenguaje científico se utiliza 
para expresar ideas en secuencia lógica que se unen a través de aparatos lingüísticos. Algunos ejemplos son: repetir palabras, parafrasear, usar vocablos semánticos similares, usar conectores de lógica (porque, por lo tanto, consecuentemente, pero, por ejemplo).

El estudiante de ciencia también tiene que desarrollar un vocabulario científico que es específico. El idioma y los conceptos se deben presentar simultáneamente dentro de un contexto comunicativo en donde el proceso cognoscitivo para adquirir el lenguaje resulte de los efuerzos comunicativos al llevar a cabo tareas de ciencia aplicada. La enseñanza de ciencia a través de la investigación y la solución de problemas enfatiza unos procesos para confrontar situaciones: definir el problema, establecer suposiciones, recoger y analizar información, y hacer generalizaciones. La enseñanza de un segundo idioma a través de la ciencia facilita el que los estudiantes se involucren en la investigación y en el análisis de problemas con significado académico que estimulan a su vez los procesos inherentes de pensamiento y análisis, tales como: observar, clasificar, comparar, medir, inferir, predecir, comunicar y establecer relaciones de tiempo y espacio. Este enfoque pedagógico promulga el aprendizaje del lenguaje dentro de un contexto comunicativo con el propósito de transmitir conceptos y establecer relaciones de ciencia que son relevantes para el estudiante. La enseñanza del segundo idioma mediante la ciencia se lleva a cabo a través de procesos cognoscitivos, tales como, observación, inferencia, predicción, hipótesis y experimentación. El segundo idioma se utiliza para: describir relaciones, escribir informes, seleccionar y establecer orden, indicar posibles soluciones, clasificar información, hacer preguntas, crear respuestas tentativas, hacer predicciones, evaluar evidencia para apoyar o rechazar soluciones, defender una interpretación y hasta para diseñar actividades de seguimiento que puedan contestar nuevos interrogantes.

Las actividades para enseñar el segundo idioma a través de la ciencia también crean estructuras que promueven la interacción socioacadémica entre los estudiantes y por lo tanto, proveen un contexto apropiado para la adquisición del idioma. Fillmore (I982) establece que la adquisición del segundo idioma requiere una estructura que agrupe a los estudiantes con más dominio del segundo idioma con 
los estudiantes que no dominan este idioma. Tal estructura promueve un contexto académico que asegura la interacción entre una persona que no domina el segundo idioma con una persona con dominio del segundo idioma y que toma en cuenta las limitaciones del aprendiz con menos dominio del segundo idioma. Esta interacción es aún más efectiva si se lleva a cabo a través de actividades que sean relevantes y culturalmente apropiadas. Un grupo de estudiantes con habilidades y experiencias diferentes ejecutando una tarea en ciencias resultará en una comunidad de aprendices con diversos esquemas culturales, sociolingüísticos y académicos. Estos diversos esquemas pueden causar que los estudiantes procesen la adquisición tanto de las ciencias como del segundo idioma en formas diferentes. Para armonizar las diferencias es necesario que las actividades de enseñanza provean avenidas para que los estudiantes establezcan puentes entres los diferentes esquemas de tal forma que se facilite el aprendizaje del contenido científico y la adquisición del segundo idioma. En la mayoría de los casos las estructuras mentales, tanto de los conceptos de la materia como de idioma, tienen que ser modificadas. Las actividades que promueven la interacción entre los alumnos y el uso de manipulativos ayudan a reestructurar tanto las percepciones del mundo físico como las formas y procesos de pensamiento y a la vez proveen un ambiente apropiado para el desarrollo del segundo idioma.

\section{Guía Integrativa para la Enseñanza de un segundo idioma a través de la Ciencia}

Los siguientes pasos se deben tomar en consideración al desarrollar una unidad curricular para enseñar el inglés como segundo idioma mediante la ciencia o las matemáticas.

\section{Establezca un marco conceptual para fundamentar su unidad curricular}

I. Utilice la literatura y las teorías presentadas en los currículos regulares de la materia y del inglés como segundo idioma. El marco conceptual se utiliza para:

a. ayudar a respaldar sus creencias sobre el aprendizaje y el desarrollo de un currículo,

b. proveer legitimidad, 
c. presentar los paradigmas de otros expertos que respaldan sus creencias,

d. sentar las bases para la descriminación necesaria para poder seleccionar las actividades y los materiales apropiados y los que concuerdan con la vision y filosofía de la enseñanza compartida por los autores del currículo,

e. guiar el desarrollo de los objetivos,

f. ayudar a determinar la forma y el alcance filosófico y estructural de las modificaciones a llevarse a cabo en el currículo actual,

g. exponer los prejuicios educativos de los autores.

\section{Determine las necesidades de los estudiantes.}

I. Conduzca un estudio para determinar las necesidades lingüísticoacadémicas y el ambiente educativo de los estudiantes.

2. Recoja información utilizando tanto instrumentos estandarizados como procesos alternativos para generar datos. El estudio de las necesidades requiere que se:

a. identifique los exámenes que usa el distrito escolar para medir el aprovechamiento académico de los estudiantes en el primer y en el segundo idioma, en ciencia o en matemáticas;

b. identifique el trasfondo socio-cultural de los estudiantes;

c. examine la información generada a través del cuestionario de idioma completado por los padres;

d. examine el informe escolar de los estudiantes para recaudar información relevante sobre el estudiante;

e. conduzca entrevistas con los maestros y otros miembros del personal escolar para acumular información sobre las necesidades de los estudiantes;

f. desarrolle un perfil lingüístico-académico de los estudiantes con la información generada a través de los instrumentos y los procesos antes mencionados;

g. estudie los mandatos del distrito escolar relacionados con la enseñanza de las materias y del segundo idioma y el desarrollo de un currículo;

h. identifique y describa el ambiente comunicativo del estudiante en la escuela y el hogar; 
i. determine la opinión que los padres tienen respecto a su proyecto.

\section{Promueva la colaboración.}

\section{Consulte y trabaje en colaboración con el personal educativo y con los representantes de los padres.}

I. La colaboración entre los maestros de las materias y los de la clase del segundo idioma permite que el proyecto incorpore el conocimiento de expertos en las materias académicas y de expertos del segundo idioma. El equipo del personal que va a colaborar en el desarrollo del currículo debe:

a. incluir personal que favorezca y se comprometa con el proyecto, a la vez que comparta similares creencias educativas,

b. asegurar el tipo de colaboración que promueva las metas y evite la duplicación,

c. consolidar los recursos, reunir los expertos e integrar las materias,

d. incluir al principal o director de la escuela y al coordinador de currículo,

e. incluir personal que represente puntos de vista diferentes en relación a su filosofía educativa para enriquecer y expandir sus conocimientos,

f. establecer el plan de trabajo con los procedimientos para llevar a cabo las tareas,

g. establecer el proceso de monitoría,

h. desarrollar un calendario con las fechas para completar las tareas.

\section{Selección de la materia y el contenido.}

A. Escoja e incorpore una sola materia a la enseñanza del segundo idioma. Gradualmente, y después que demuestre el éxito del proyecto, se puede incorporar una segunda materia.

I. Seleccione una unidad del currículo regular que sea de interés para los estudiantes y que sea suceptible a la enseñanza de un segundo idioma. Determine:

a. el respaldo e interés del personal, 
b. el nivel del previo conocimiento del contenido que se requeriere para que los estudiantes entiendan los conceptos,

c. la dependencia entre el contenido y el idioma,

d. el nivel de proficiencia de idioma requerido para el entendimiento de los conceptos básicos,

e. los conceptos y temas más esenciales de la materia,

f. los procesos de aprendizaje que puedan ser utilizados en la instrucción tanto de la materia como del segundo idioma,

g. cómo va a dividir los temas en subtópicos que sean adecuados para la enseñanza del segundo idioma,

h. qué temas se dirigen a las destrezas de pensamiento que desea enfatizar y cuáles son los niveles de dificultad de estas destrezas,

i. la calidad y la cantidad de los materiales disponibles para llevar a cabo actividades apropiadas.

\section{Organización de la unidad.}

I. Una unidad curricular debe incluir, por lo menos, las siguientes partes: el marco conceptual, la descripción del ambiente educativo y las necesidades del estudiante, los planes de lecciones diarias o de periodos pre-determinados, una bibliografía, y un apéndice.

2. El marco conceptual debe preceder todas las otras partes de la unidad. Se debe escribir en una forma simple y correcta, y solo debe incluir referencias que aparecen en la bibliografía.

3. La descripción del ambiente educativo y las necesidades del estudiante debe estar basada tanto en información cuantitativa como cualitativa. La infomación sobre el ambiente educativo debe:

a. enfatizar las condiciones que tienen impacto en la instrucción y la enseñanza,

b. describir tanto el ambiente particular de la escuela, como los elementos del currículo y de la política del distrito que se relacionen con el desarrollo curricular,

c. incluir las condiciones educativas en el hogar y en el vecindario. 
La información sobre el estudiante debe:
a. identificar la edad y el grado del estudiante,
b. describir su nivel de proficiencia en ambos idiomas,
c. describir su nivel académico en la materia(s) seleccionada(s),
d. presentar sus experiencias académicas, culturales o extracurriculares que se relacionan con el contenido, idioma o destrezas que se desean desarrollar,
e. discutir condiciones o necesidades especiales que tenga,
f. presentar toda otra información sobre el estudiante que sea relevante y que ayude a los maestros a diseñar actividades para asistir a los estudiantes a sobrepasar necesidades particulares y para facilitarle el aprendizaje.

4. Los planes de las lecciones diarias son unas guías que aseguran que se alcancen los objetivos de corto y largo plazo. Los planes pueden requerir uno o más períodos de clase o menos tiempo.

5. Las partes primordiales de los planes son los objetivos, las actividades, los materiales, y la identificación y evaluación de las destrezas que el aprendiz va a desarrollar.
a. los objetivos de los planes diarios tienen que estar en concordancia con las metas o los objetivos generales de la unidad,
b. las actividades tienen que llevarse a cabo de tal forma que alcancen los objetivos establecidos,
c. las actividades tienen que facilitar el desarrollo de las destrezas que el estudiante necesita adquirir,
d. los materiales necesitan incentivar el desarrollo de las destrezas,
e. la evaluación del aprendizaje debe ser congruente con los objetivos establecidos.

6. Las destrezas que el aprendiz va a desarrollar tienen que ser identificadas en forma clara y precisa. Estas destrezas:

a. tienen que estar enfatizadas en los objetivos establecidos,

b. determinan el tipo de actividades que se necesitan para que el estudiante pueda participar en el proceso de enseñanza y aprendizaje, 
c. son las que se enfatizan en la evaluación del aprendizaje.

7. Desarrolle objetivos congruentes con el marco conceptual.

I. La unidad curricular debe establecer metas u objetivos generales y objetivos diarios o para cada período que la lección abarca.

Los objetivos generales deben corresponder a las metas $\mathrm{u}$ objetivos del currículo regular. Los objetivos diarios deben corresponder tanto a:

2. Los objetivos generales del currículo regular como a los de la unidad.

3. Los objetivos de contenido deben corresponder y suplementar los objetivos de contenido de la materia del currículo regular. Los objetivos de contenido deben:

4. dirigirse hacia el desarrollo de los conceptos del contenido de la materia,

5. incluir diferentes niveles de pensamiento,

6. enfatizar destrezas complejas de pensamiento tanto en la materia como el idioma,

7. dirigirse a las necesidades lingüísticas y académicas del estudiante,

8. ser formulados de tal forma que se puedan medir cuantitativa y cualitativamente,

9. enfatizar la aplicación de los conceptos aprendidos,

I0. establecer los procesos de aprendizaje,

II. establecer las estrategias de aprendizaje (por ejemplo: el uso de material gráfico, el reconocimiento de las partes del libro, la identificación de las fuentes de información, la reoganización de páragrafos, y la organización de la información).

I2. Los objetivos específicos del segundo idioma deben relacionar el aprendizaje del idioma con el aprendizaje de contenido. Los objetivos deben:

a. reflejar el nivel de idioma del contenido en el currículo regular,

b. estar basados en las necesidades lingüísticas y académicas del estudiante, 
c. estimular la adquisición del vocabulario técnico incluido en el contenido,

d. incentivar el aprendizaje del lenguaje necesario para el entendimiento de la materia (lógica, relaciones, funciones, significados, símbolos, etc.),

e. enfatizar el uso del idioma como medio de aprendizaje del contenido,

f. incluir las cuatro áreas de destrezas del lenguaje (leer, escribir, escuchar y hablar) a pesar de la diversidad de los niveles de proficiencia del segundo idioma,

g. enfocar el desarrollo de actividades interactivas,

h. impulsar la comunicación con propósitos académicos entre los estudiantes,

i. requerir el uso del segundo idioma con propósitos académicos (lectura crítica y evaluativa, reflexión de pensamiento y expresión de ideas a través del habla y la escritura),

j. enfatizar las destrezas de lenguaje entrelazadas con las destrezas de pensamiento analítico tales como clasificación (definir y categorizar), los principios (explicar y predecir: interpretar, teorizar, generalizar-causa, efecto, motivo, razones, reglas, normas, resultados, impacto, relaciones, símbolos), y la evaluación (rango, juicio, preferencia, selección, alternativas, criterio),

k. enfatizar que muchas de las destrezas de contenido, idioma y pensamiento analítico, tales como las mencionadas arriba, se pueden transferir de unas materias a otras,

1. reflejar el uso y la aceptación del idioma y de la cultura del hogar del estudiante.

Los planes de las lecciones diarias guían la instrucción del día o del periodo de clase para asegurarse que los estudiantes puedan alcanzar los objetivos establecidos tanto en la ciencia o la matemática como en el segundo idioma. 


\section{Conclusiones}

Se considera que este artículo es importante para la comunidad científica internacional que aborda el aprendizaje de un idioma extranjero como segunda lengua, porque en él se hace referencia a marcos teóricos de origen socio-cultural y lingüístico-cognoscitivo que han influido en la enseñanza de inglés como segundo idioma, se refleja la Integración del Inglés Como Segundo Idioma con la Ciencia en sentido general y se desarrolla una Guía Integrativa para la Enseñanza de un segundo idioma a través de la Ciencia. El autor piensa que la misma puede constituir un recurso valioso para el desarrollo del proceso de enseñanza y aprendizaje de cualquier idioma como segunda lengua.

\section{Referencias bibliográficas}

Bley-Vroman, R. (1988). The fundamental character of foreign language learning. In W. Rutherford \& M. Sharwood-Smith (Eds.), Grammar and second language teaching, (pp. 19-30). New York: Newbury House.

Carroll, J. (I999). The contributions of psychological theory and educational research to the teaching of foreign languages. Modern Language Journal, 49, 273-28l.

Canale, M. \& Swain, M. (1980). Theoretical bases of communicative approaches to second language teaching and testing. Applied Linguistics, l, l-47.

Chomsky, N. (1965). Aspects of the theory of syntax. Cambridge, MA: MIT Press.

Cummins, J. (1979). Linguistic interdependence and the educational development of bilingual children. Review of Educational Research, 48, 222-25l.

Cummins, J. \& Swain, M. (I995). Bilingualism in education: Aspects of theory, research and practice. London: Longman.

Fillmore, L. W. (I995). Language minority students and school participation: What kind of English is needed? Journal of Education, 164, (2), 143-156.

Harris, D.P. (1969). Testing English as a second language. New York: McGraw-Hill.

Krashen, S. (I98I). Second language acquisition and second language learning. Oxford: Pergamon.

Krashen, S. D. \& Terrell, T.D. (1983). The natural approach. Hayward: CA Alemany. 
Oller, J.W. (1979). Language tests at school. London: Longman.

Rummelhart, D. E. (1980). Schemata: The building blocks of cognition. In R. J. Shapiro, B. C. Bruce, \& W. F. Brewer (Eds.) Theoretical issues in reading comprehension (pp. 33-58). Hillsdale, NJ: Erlbaum.

Spolsky, B. (1973). What does it mean to know a language, or how do you get someone to perform his competence? In J. W. Oller, Jr. \& J. C. Richards (Eds.). Focus on the learner: Pragmatics perspectives for the language teacher ( $p p$. 164-176). Rowley, MA: Newbury.

White, L. (1990). Second language acquisition and universal grammar. Studies in Second Language Acquisition, 12 (2), I2I-I34. 